\title{
Management of Acute Facial Nerve and Parotid Injuries
}

\author{
Tyler J. McElwee, MD ${ }^{1}$ John N. Poche ${ }^{2}$ Justin C. Sowder, MD ${ }^{1,3}$ Laura T. Hetzler, MD ${ }^{3,4}$
}

${ }^{1}$ Department of Otolaryngology-Head and Neck Surgery, LSU Health Sciences Center New Orleans, Baton Rouge, Louisiana

${ }^{2}$ College of Medicine, LSU Health Sciences Center New Orleans, Baton

Rouge, Louisiana

${ }^{3}$ Department of Otolaryngology Head and Neck Surgery, Our Lady

of the Lake Regional Medical Center, Baton Rouge, Louisiana

${ }^{4}$ Department of Otolaryngology-Head and Neck Surgery, Louisiana

State University Health Sciences Center, New Orleans, Louisiana

\author{
Address for correspondence Justin C. Sowder, MD, Department of \\ Otolaryngology-Head \& Neck Surgery, LSU Health Sciences Center \\ New Orleans, 533 Bolivar St. Suite 566 Baton Rouge, LA 70809 \\ (e-mail: jcsowder@gmail.com).
}

Facial Plast Surg 2021;37:490-499.

\begin{abstract}
Keywords

- facial trauma

- facial nerve

- parotid duct

- parotid gland
\end{abstract}

Acute soft tissue injuries to the head and neck are a common reason for patients to present to the emergency department and require evaluation by a facial plastic surgeon. Facial anatomy is complex, as it houses four of the five major senses as well as speech. Our face is the primary means by which others identify us as individuals as well as how we express ourselves to the world. The cheek is the subunit of the face with the largest surface area and is frequently injured. It is also the subunit overlying the main trunk and inferior branches of the facial nerve (FN), the parotid gland, and the parotid duct (PD). While cutaneous soft tissue injuries to the cheek are easily identified on inspection, a high degree of suspicion must be maintained during the initial evaluation to determine if there has been damage to these deeper structures. Failure to identify or completely repair injuries to the FN or the parotid can lead to significant morbidity for the patient down the road.

published online April 20, 2021

\section{Traumatic Facial Nerve Paralysis Secondary to Transection}

The incidence of peripheral FN palsies ranges from 20 to 30 cases per 100,000 patients per year-one of the most common cranial nerve pathologies. While most cases are idiopathic, traumatic etiologies are responsible for up to $30 \%$ of cases, most in the form of temporal bone fractures. FN injury following soft tissue trauma usually occurs in the setting of stab wounds, gunshot wounds, or motor vehicle accidents.

\section{Anatomy}

The FN begins intracranially at the pons and then takes a complex course through the internal auditory canal into the temporal bone, where it makes two turns ("genus") before it exits the skull base at the stylomastoid foramen. Peripherally, (c) 2021. Thieme. All rights reserved. Thieme Medical Publishers, Inc., 333 Seventh Avenue, 18th Floor, New York, NY 10001, USA
DOI https://doi.org/ 10.1055/s-0041-1725129. ISSN 0736-6825. and Healing; Guest Editor: Sydney C. Butts, MD, FACS

Issue Theme Facial Soft Tissue Injury Butts, MD, FACS 
the main trunk of the extratemporal FN courses anteriorly and deep to the posterior belly of the digastric for approximately 1 to $1.5 \mathrm{~cm}$ before it splits at the pes anserinus (Latin for "goose foot") where it bifurcates into the temporofacial and cervicofacial divisions. Though the branching pattern of the distal FN is variable, the temporofacial branch typically divides into the terminal temporal (frontal) and zygomatic branches, while the lower cervicofacial branch trifurcates into the buccal, marginal mandibular, and cervical branches.

Injuries at different anatomical levels of the FN can produce different deficits. The more distal an injury, the more motor and less sensory and parasympathetic deficits. Nonmotor deficits to be evaluated include xerophthalmia, nasal dryness, dysgeusia or ageusia, and xerostomia.

\section{Evaluation}

The evaluation of a facial trauma victim should always include a complete cranial nerve exam. Assessment of the FN should be done in a systematic fashion, as particular deficits can elucidate the branches compromised. This should always be done prior to the injection of local anesthetic. The face is typically divided into five motor segments assessed in a superior to inferior direction: forehead, periocular, midface, perioral, and cervical. Assessment of the forehead involves brow elevation and brow furrowing. Periocular evaluation focuses heavily on gentle and forceful eye closure to assess all muscular components of the orbicularis oculi. Lower eyelid position and tension is noted, but can be affected by midfacial function and ptosis as well. Midfacial function is assessed with wrinkling of the nose and smiling or lip elevation. The perioral function is evaluated by pucker and pouting motions and the cervical division by flexing of the platysma. ${ }^{1}$ Areas of obvious trauma can quickly be assessed with their relation to the lateral canthus. If the injury is medial to the lateral canthus, immediate repair may not be warranted as the nerve is more likely to regain function without surgical management due to extensive cross innervation. A distinction should be made between paresis and paralysis, as paretic muscles imply continuity of the nerve and such injuries can be managed conservatively. ${ }^{2}$

\section{Timing of Exploration}

The presence of complete paralysis at the time of evaluation portends a poor prognosis as compared with delayed paralysis. In such scenarios, the timing of exploration and repair has consistently been the largest predictive factor to successful outcomes in traumatic FN transection. Exploration should occur within 72 hours of injury to allow electrical stimulation of distal branches to facilitate identification, otherwise Wallerian degeneration would progress to where stimulation is not feasible. Electroneuronography can be useful between 3 and 21 days after injury but starting at 3 weeks electromyography (EMG) is the gold standard to determine the potential for reinnervation. EMG begins to demonstrate fibrillation potentials 2 to 3 weeks after denervation of the facial musculature, and subsequent polyphasic action potentials (PAPs) indicate regeneration is occurring. If PAPs are present, this may indicate a higher likelihood of spontaneous return of motor function, allowing for more conservative management. Absence of fibrillation potentials and PAP's indicates irreversible fibrosis. During the first 3 months after injury, contraction secondary to scarring reduces the cross-sectional area of the nerve up to $70 \%{ }^{3}$

In the cases of either delayed diagnosis or delayed intervention due to the patients' instability, there is no definitive agreement regarding the optimal time for reinnervation procedures, though it is widely believed that the earliest repair possible results in the greatest functional outcome. Immediate direct neurorrhaphy has shown the best functional results. The current literature is contradictory, with some studies supporting better functional outcomes when repaired within 90 to 180 days, while others comparing immediate and early repair, defined as 6 to 18 months post injury, have demonstrated comparable outcomes. The latter study did show that nerve function returns significantly faster in the early repair arms. ${ }^{4-6}$ There is a large consensus that the best possible functional outcome after complete transection of the FN, regardless of the method of coaptation and time until intervention, is HouseBrackmann grade III. ${ }^{6}$

\section{Nerve Repair Techniques}

\section{Direct Neurorrhaphy}

Direct neurorrhaphy is the gold standard for FN repair. However, this technique requires a tension free neurorrhaphy of the severed nerve ends, which is not always possible. If tension is present at this site, impaired vascular flow may lead to scarring and inhibit axonal regeneration. Attempts can be made to mobilize the nerve inferiorly and superiorly to ease tension.

The neurorrhaphy itself is typically performed using an end-to-end coaptation following conservative trimming of the nerve ends. A cuff of epineurium is retracted away from the cut end to allow the fascicles to be trimmed sharply. The epineurium is then released to overlap the trimmed fascicles slightly, allowing the fascicles to lay gently end-to-end once the epineurium is approximated. The coaptation is done with 9-0 or 10-0 nylon sutures through the epineurium under an operating microscope (-Fig. 1). Direct neurorrhaphy results in the best outcomes compared with other techniques in terms of both a faster and more significant improvement in House-Brackmann scores. ${ }^{5}$

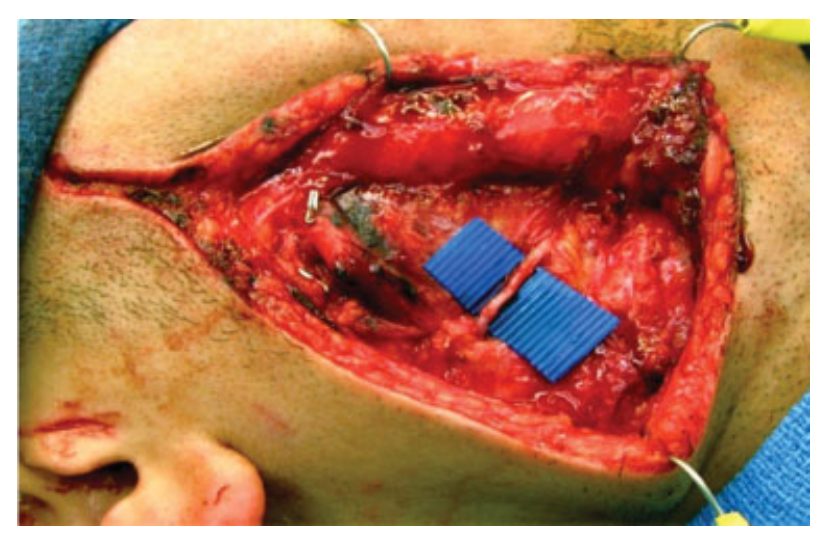

Fig. 1 Intraoperative view following primary neurorrhaphy of transected facial nerve due to a penetrating cheek injury. 


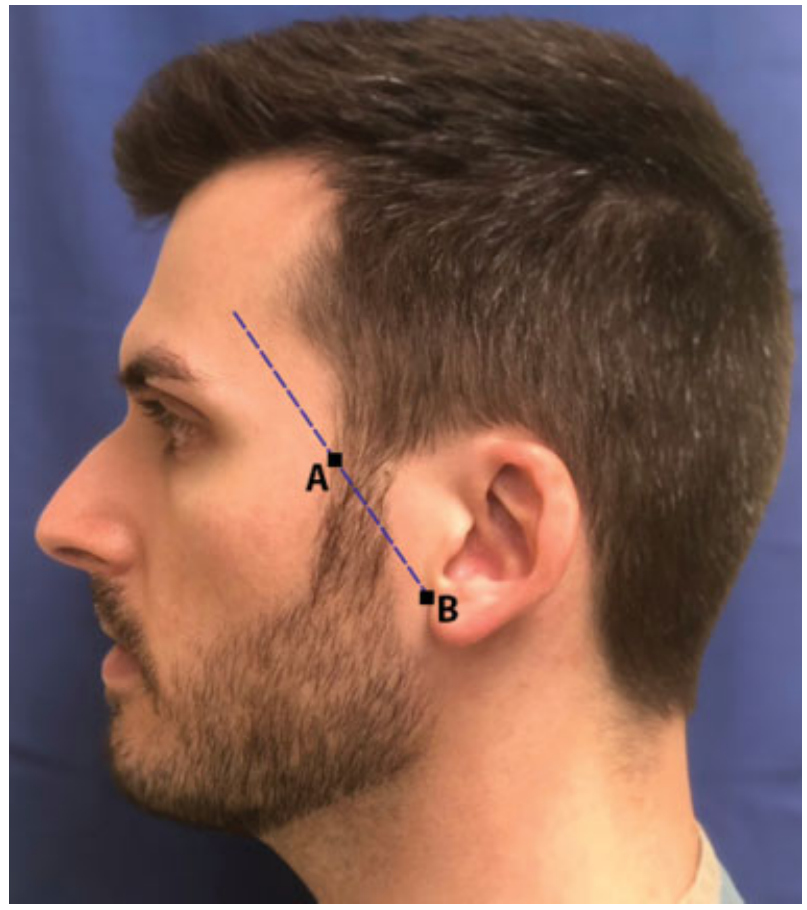

Fig. 2 The path of the frontal branch of the facial nerve can be estimated by Pitanguy's line, which is line drawn through two points: (A) midpoint between the root of the helix and the lateral canthus and (B) the root of the ear lobule.

Difficulty identifying the proximal and distal ends of the FN in the setting of robust soft tissue injury can be problematic. Identifying the main trunk of the FN either within the mastoid or utilizing the reliable landmarks at its exit from the stylomastoid foramen may be helpful. Distal identification may require identification of peripheral branches with retrograde dissection toward the main trunk.

Peripherally, certain anatomical landmarks are helpful to approximate the location of each branch of the FN. ${ }^{7}$

\section{Frontotemporal Branch}

The path can be estimated by Pitanguy's line (-Fig. 2), defined as a line drawn from $0.5 \mathrm{~cm}$ inferior to the tragus to a point $1.5 \mathrm{~cm}$ superior and lateral to the eyebrow.

\section{Buccal Branch}

It is located by its proximity to the PD, typically running within $1 \mathrm{~cm}$ inferior to the duct and along the same trajectory.

\section{Marginal Mandibular Branch}

Typically runs 1 to $2 \mathrm{~cm}$ inferior to the inferior border of the angle of the mandible, however, it can be found up to $3 \mathrm{~cm}$ inferiorly.

\section{Cervical Branch}

It is found that approximately $1 \mathrm{~cm}$ posterior to the gonion. Incisions $2 \mathrm{~cm}$ posterior to the gonion will typically preserve the inferior parotid pole and lower branches of the FN. Another approximation is a line drawn from the mentum to the mastoid process, with a perpendicular line $1 \mathrm{~cm}$ inferior to the angle of the mandible estimating the location of the nerve., ${ }^{7,8}$

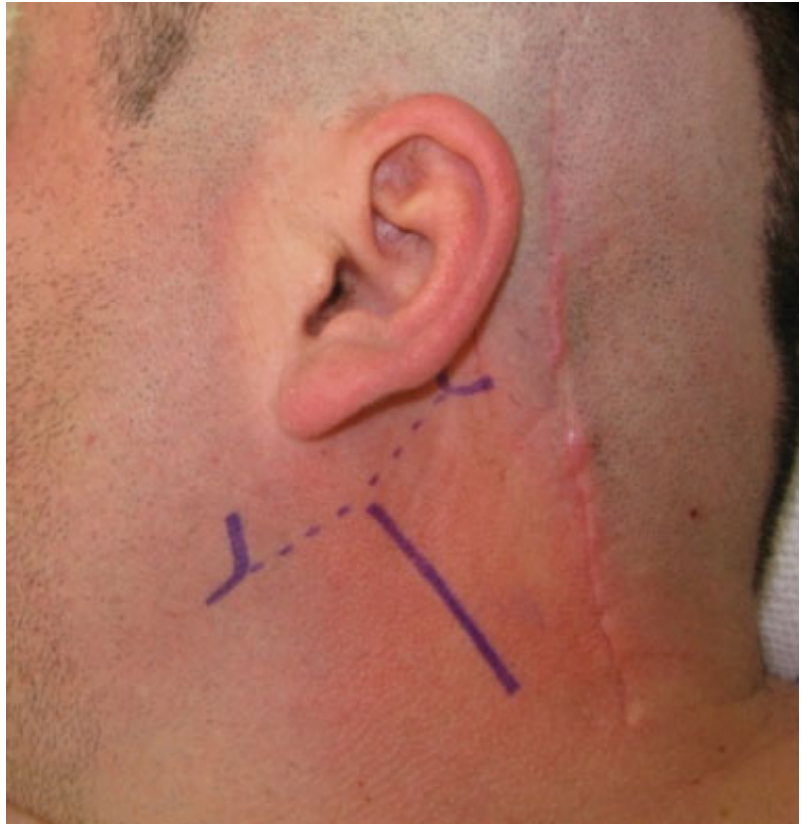

Fig. 3 The great auricular nerve is identified by perpendicularly bisecting a line drawn from the angle of the mandible to the mastoid tip over the sternocleidomastoid.

\section{Interposition Nerve Grafting}

If a tension free direct neurorrhaphy is not possible, an autograft or allograft can be used to bridge the gap and provide a tension free neurorrhaphy. Common autograft donor sites include the great auricular nerve (GAN), sural nerve (SN), and the medial antebrachial cutaneous (MABC) nerves.

\section{Great Auricular Nerve}

The GAN is utilized most frequently given its proximity, roughly equivalent cross-sectional area, and low donor site morbidity. The GAN can typically provide 6 to $8 \mathrm{~cm}$ in length. ${ }^{3}$ It is found approximately $6.5 \mathrm{~cm}$ below the inferior wall of the external acoustic meatus and $0.5 \mathrm{~cm}$ above the external jugular vein. ${ }^{4}$ Another method of identification is with a line drawn from the angle of the mandible to the mastoid tip, with the GAN bisecting this line as it passes over the sternocleidomastoid. ${ }^{9}$ It runs deep to the platysma muscle in the same general course of the external jugular vein (-Fig. 3 ).

\section{Sural Nerve}

The $\mathrm{SN}$ is harvested when length is a concern or there is a need to graft multiple distal nerve branches. The SN approximates the cross-sectional area of the FN and can supply up to $40 \mathrm{~cm}$ of graft. $^{3}$ It is found as it leaves the tibial nerve in the popliteal fossa and descends between the two heads of the gastrocnemius, piercing the deep fascia at the posterior aspect of the lower leg. It is often just posterior to the lateral malleolus and can be harvested with either one long incision, several transverse "stair-step" incisions, or endoscopically. For open harvest, the first incision is made $2 \mathrm{~cm}$ posterior and $2 \mathrm{~cm}$ proximal to the lateral malleolus, as the nerve has not undergone significant branching at this point (-Fig. 4). If the small saphenous vein is encountered, the nerve lies just medial to it. A tendon stripper may be useful to clear off the soft tissue. For 


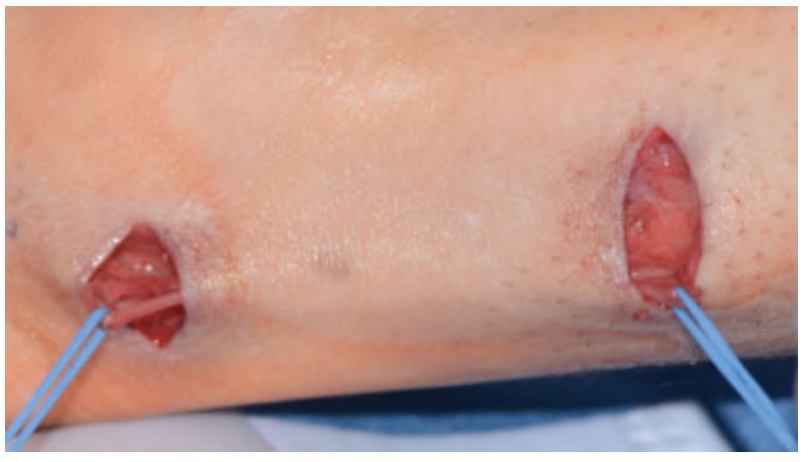

Fig. 4 Left sural nerve harvested through stair-step incisions with resulting graft.

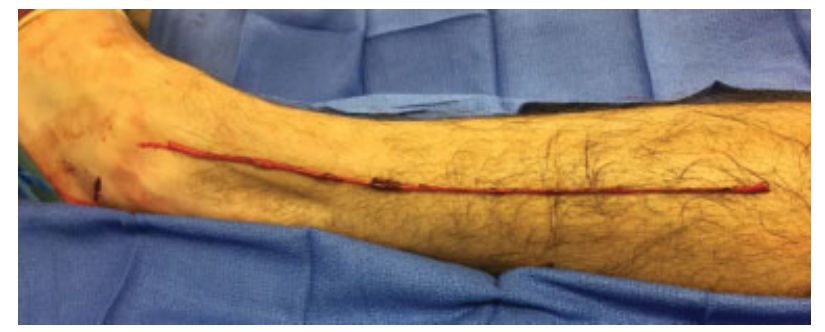

Fig. 5 Left sural nerve graft after harvest through stair step incisions

endoscopic harvest of the $\mathrm{SN}$, a horizontal incision is made approximately $1 \mathrm{~cm}$ behind the lateral malleolus. The saphenous vein is identified, and the SN identified immediately posterior to this. The endoscope and endoharvester are passed along the length of the nerve to skeletonize the required length. The nerve is then cut proximally and delivered from the inferior incision ( - Fig. 5 ). ${ }^{10}$ The disadvantages to using the $\mathrm{SN}$ as an autograft are the need for another operative site, scarring, and sensory deficits of the lateral foot.

\section{Medial Antebrachial Cutaneous Nerve}

The MABC nerve offers an ideal size match and branching pattern as an autologous donor nerve. This nerve can be used when repairing defects extending from the main trunk or intra-temporal nerve to multiple distal branches. A cutaneous incision is placed along the medial bicipital groove (-Fig. 6). The MABC nerve is located near the cephalic vein in the superior forearm, and can be found as it emerges laterally from the inferior aspect of the biceps brachii tendon as it pierces the inter-epicondylar line. At this level, it is approximately $1 \mathrm{~cm}$ medial to the cephalic vein and can be seen decussating to innervate the forearm $\operatorname{skin}^{11}$ ( - Figs. 7 and $\mathbf{8}$ ).

\section{Polarity of Grafts}

Polarity is a commonly debated topic when grafting nerves. Some advocate reversing an autograft's polarity under the belief that it reduces aberrant re-arborization of nerve fibers. Multiple animal studies have demonstrated that reversing the polarity of an autograft did not have significant effects on the total number of axons regenerated, nerve conduction velocity, or functional outcomes. ${ }^{12}$ More consideration should be given to nerve diameter matchup than polarity in optimal nerve reconstruction.

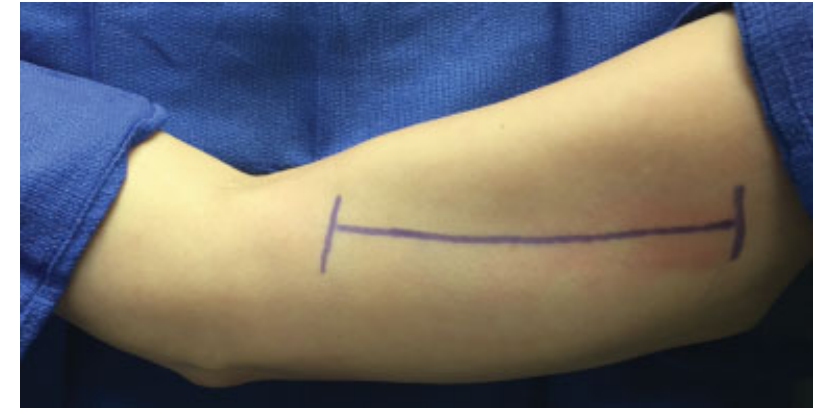

Fig. 6 Median antebrachial cutaneous nerve harvest. Incision marked in the right bicipital groove

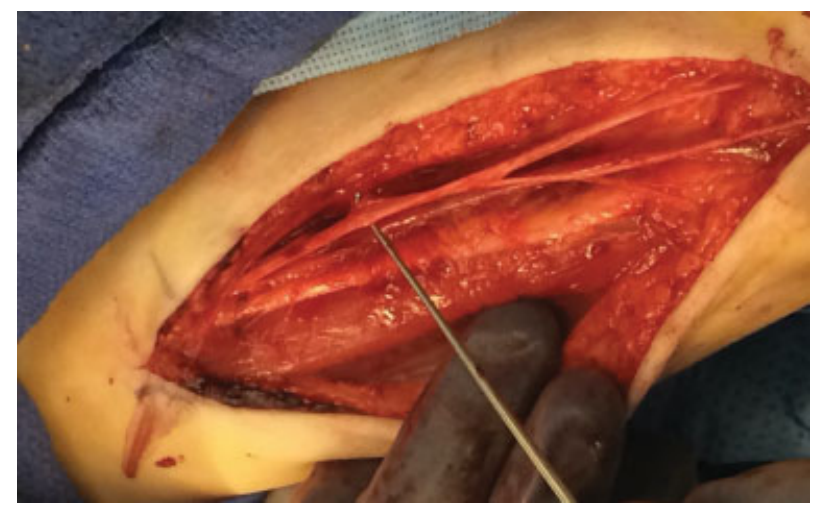

Fig. 7 The median antebrachial cutaneous nerve as it emerges laterally from the inferior aspect of the biceps brachii tendon as it pierces the inter-epicondylar line.

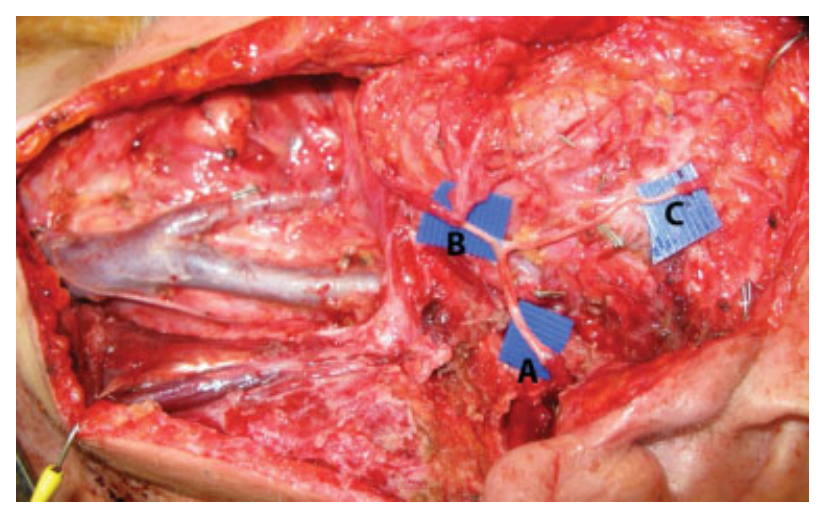

Fig. 8 Median antebrachial cutaneous nerve interposition graft spanning a gap in the facial nerve at the pes anserinus from the main trunk (A) to the cervicofacial (B) and temporofacial (C) divisions.

\section{Nerve Allografts}

If the patient's GAN, SN, or MABC nerves are either unavailable or unable to be used, an allograft can be used to bridge the gap and provide a tension free repair. Situations in which an allograft may be preferred over an autograft are patients who have received radiation at the donor and/or recipient site, tumor invasion, or significant comorbidities precluding prolonged operative times. A major benefit of allografts is the lack of donor site morbidity. Traditional allografts have fallen out of favor due to the requirement for long-term host immunosuppression, leading to the development of decellularized nerve allografts. $^{13}$ These allografts have reduced 


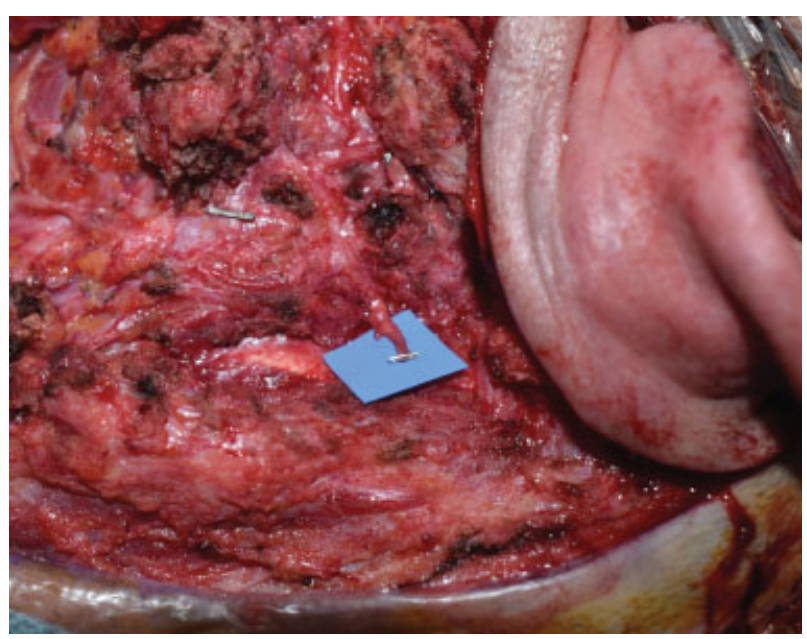

Fig. 9 Proximal stump of the main trunk of the left facial nerve following traumatic transection with segment missing.

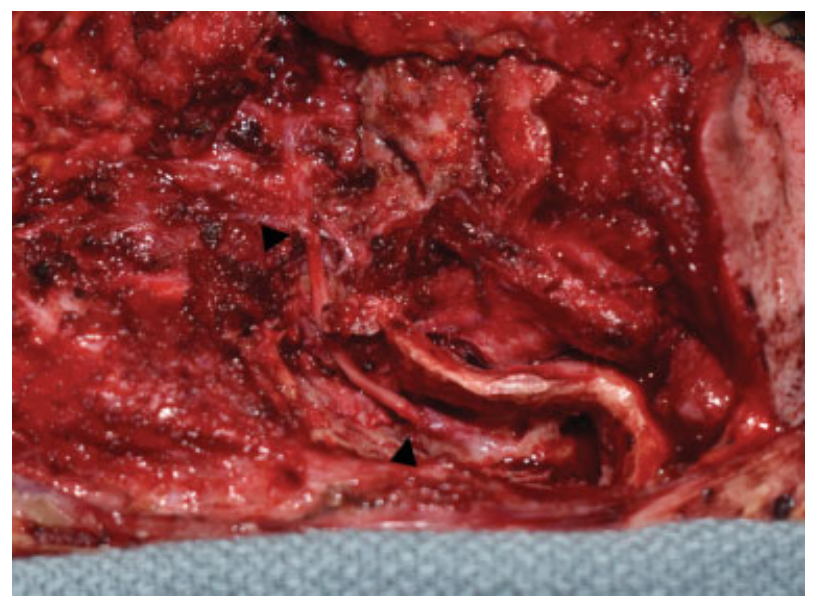

Fig. 10 Avance nerve allograft (Axogen; Alachua, FL) used as interposition graft between the mastoid segment and the proximal trunk of the traumatically transected facial nerve.

immunogenicity but retain the beneficial characteristics of scaffolding and extracellular proteins that may facilitate nerve regeneration. ${ }^{14}$ Allografts performed for peripheral nerve repair support meaningful recovery of up to $89 \%$ in sensory nerves and $86 \%$ in motor nerves. ${ }^{15}$ While the majority of studies center around neurorrhaphy in the hand, recent data from reinnervation of the lingual and inferior alveolar nerves have demonstrated upward of $94 \%$ functional sensory recovery at 1 year postoperatively. ${ }^{12}$ The limited data regarding allografts in FN repair reveals suboptimal outcomes compared with direct neurorrhaphy, but allografts are still a feasible choice when an autograft is not an option (-Figs. 9 and 10). ${ }^{16}$

\section{Complications and Management}

Complications related to traumatic FN paralysis can occur as direct sequelae from the paralysis or indirectly related to the neurorrhaphy. Immediate complications related to nerve paralysis are due to the loss of both motor function and static muscle tone of the mimetic muscles of the face. A complete discussion regarding management of such complications is outside the scope of this chapter, but several of the more serious acute issues warrant mention. Loss of the upper branches of the FN can result in paralytic lagophthalmos. Corneal protection can be compromised with inability to close the eye resulting in corneal abrasions and ulcerations. Patients who suffer a concomitant injury to the ophthalmic division of the trigeminal nerve (V1) are at even higher risk of neurotropic keratopathy due to an insensate cornea. Immediate treatment of paralytic lagophthalmos consists of lubricating eye drops and a moisture chamber to mimic the physiologic function of a closed eyelid, with a low threshold to perform upper eyelid weight implantation or lateral tarsorrhaphy if the patient develops ocular pain or corneal irritation.

Complications related to neurorrhaphy include synkinesis, donor site morbidity such as pain, scarring, wound complications, and sensory deficits, and failure of meaningful functional recovery.

\section{Synkinesis or Nonflaccid Facial Paralysis}

Synkinesis is the involuntary contraction of a muscle associated with the voluntary contraction of another muscle. Synkinesis is thought to be secondary to aberrant axonal regrowth where the axons do not attach back to their original fascicles. ${ }^{3,17}$ When synkinesis occurs, it can begin as soon as 3 to 4 months after injury and can be quite distressing. The most common types of facial synkinesis include oral-ocular (involuntary eye closure during voluntary mouth movements) and ocular-oral (involuntary mouth movement during voluntary eye closure). The goals of treatment include improving resting oral commissure position, oral competence, eye closure, smile symmetry, and mimetic movement spontaneity. ${ }^{17}$ Treatment strategies include physiotherapy by an FN trained physical or occupational therapist, botulinum toxin injections, and surgical correction or augmentation. Physiotherapy aims to maximize voluntary control of the FN and associated mimetic muscles through exercise with the ultimate goal being to retrain the nerve to its proper functional pathways. ${ }^{18}$ Physiotherapy has shown to be effective in both therapy of active synkinesis as well as preventing nerve dysfunction following injuries. Botulinum toxin injections are best utilized as an adjuvant to physiotherapy. The goal is to selectively inhibit muscle contractibility of the affected areas. These are most commonly the orbicularis oculi, depressor anguli oris, mentalis, and the platysma. Choi et al demonstrated that when employed as an adjuvant treatment with physiotherapy, patients exhibited a better quality of life and improved appearance. ${ }^{19}$ Surgical treatment involves static correction of muscle hypofunction such as with brow lifting, or more dynamic effects with myectomies and selective neurolysis. ${ }^{18}$

\section{Management of Acute Parotid Gland and Parotid Duct Injuries}

\section{Epidemiology}

Parotid injuries typically occur following penetrating trauma to the cheek and are associated with damage to the adjacent 
facial structures, such as the facial and lingual nerves, the ear, and bony structures of the face. ${ }^{20}$ PD injuries account for approximately $0.21 \%$ of all trauma cases. ${ }^{21}$ Parotid injuries are more common in males and have the greatest incidence in the third decade of life. ${ }^{21}$ While approximately half of these cases are recognized as acute parotid trauma, the rest present as complications of an undiagnosed injury, such as sialoceles or fistulas. ${ }^{22}$

\section{Anatomy}

The parotid gland overlies the ramus of the mandible and is bounded superiorly by the zygoma, anteriorly by the masseter, and posteriorly by the external auditory canal and the superior aspect of the sternocleidomastoid. The gland is divided into superficial and deep portions by the FN anatomically, and the retromandibular vein radiologically. As the PD emerges anteriorly from the parotid gland, it travels superficial to the masseter in close relation to the transverse facial artery and buccal branch of the FN. ${ }^{23}$ At the anterior border of the masseter, the duct penetrates the buccinator muscle (the "masseteric bend") and oral mucosa before terminating at the level of the second maxillary molar. ${ }^{24}$ The path of the PD can be envisioned as following an "S-shaped" path that crosses an imaginary line which joins the tragus-antitragus point to the middle of the upper lip ( - Fig. 11) ${ }^{25}$ Injuries involving this area must be carefully assessed for ductal trauma.

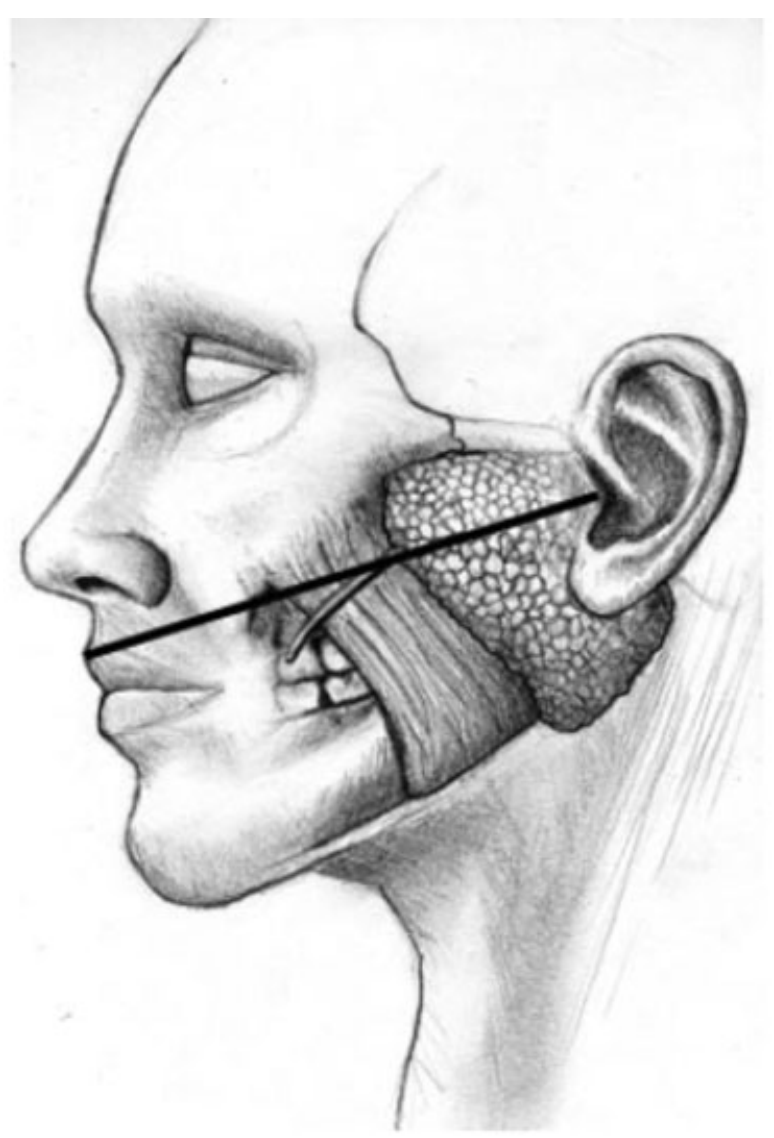

Fig. 11 The path of the parotid duct can be estimated by an "S-shaped" path that crosses an imaginary line drawn between the tragal point to the midline of the upper lip. (Reproduced with permission of Gordin et $\mathrm{al}^{36}$ ).

\section{Evaluation}

Any penetrating injury or deep laceration of the cheek along a line drawn from the tragus to the midportion of the upper lip has the potential to damage the parotid gland and/or the PD. The classification of parotid gland and ductal injuries according to location was described by Van Sickels as injuries posterior to the masseter (region A), injuries to the masseter (region $B$ ), and injuries anterior to the masseter (region $C$ ) (-Fig. 12; - Table 1) ${ }^{20}$ Region A injuries occur in the gland only, region $B$ corresponds to the duct as it runs superficial to the masseter, and region $C$ corresponds to the duct anterior to the masseter as it passes through the buccal space and attaches to the buccal mucosa opposite to the second maxillary molar. It is important to remember that the buccal branch of the FN parallels the PD, and concomitant injuries can occur in regions $B$ and C (-Fig. 13). When an injury to the gland or duct is suspected, most clinicians advocate for repair within 24 hours after the injury prior to edema making ductal cannulation more tedious. ${ }^{22}$

\section{Management}

\section{Parotid Gland Injury}

Isolated injury to the parotid gland (region A) is best treated with direct suturing of the enveloping fascia followed by application of a pressure dressing for 48 hours to reduce the

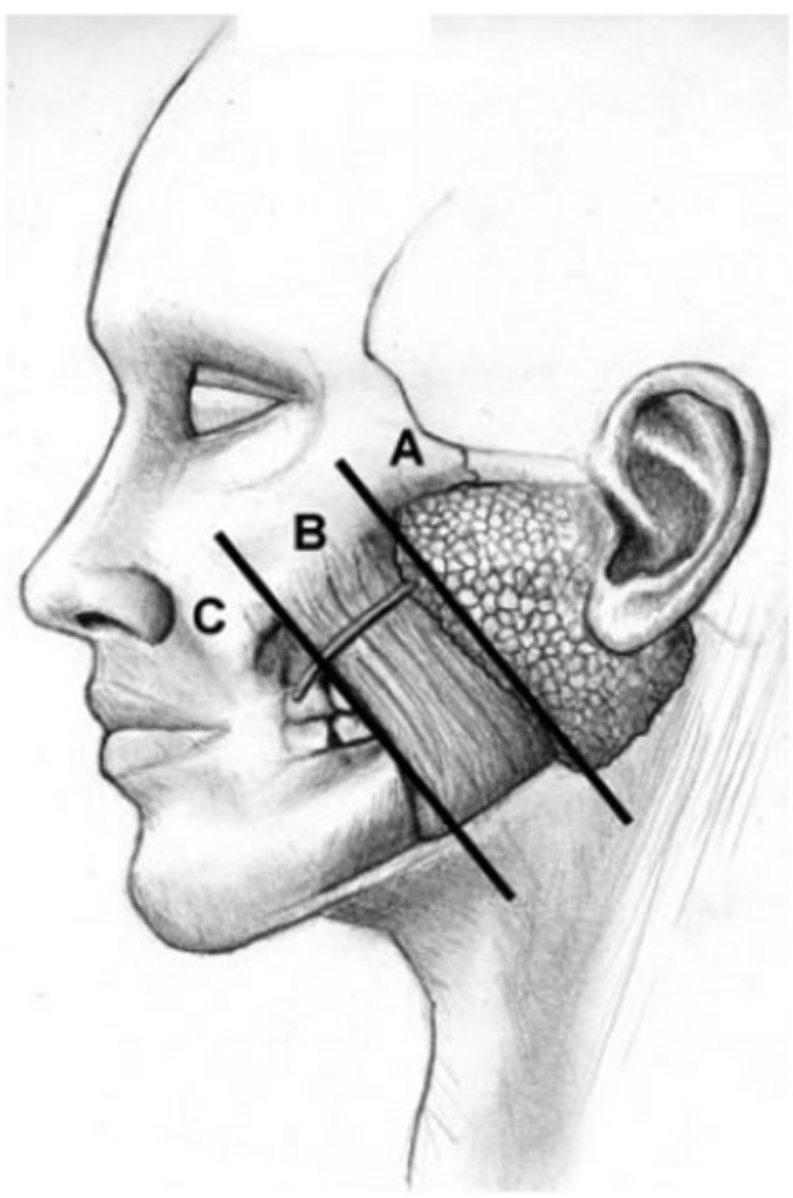

Fig. 12 Regions of injury important in parotid trauma as related to the masseter muscle. (Reproduced with permission of Gordin et $\mathrm{al}^{36}$ ). 
Table 1 Regions of parotid injury and associated complication rates

\begin{tabular}{|l|l|l|}
\hline Region of injury & Relation to masseter & $\begin{array}{l}\text { Complication } \\
\text { rate (\%) }\end{array}$ \\
\hline A & Posterior & 22 \\
\hline B & Overlying & 80 \\
\hline C & Anterior & 80 \\
\hline
\end{tabular}

Source: Adapted from Gordin et al. ${ }^{36}$

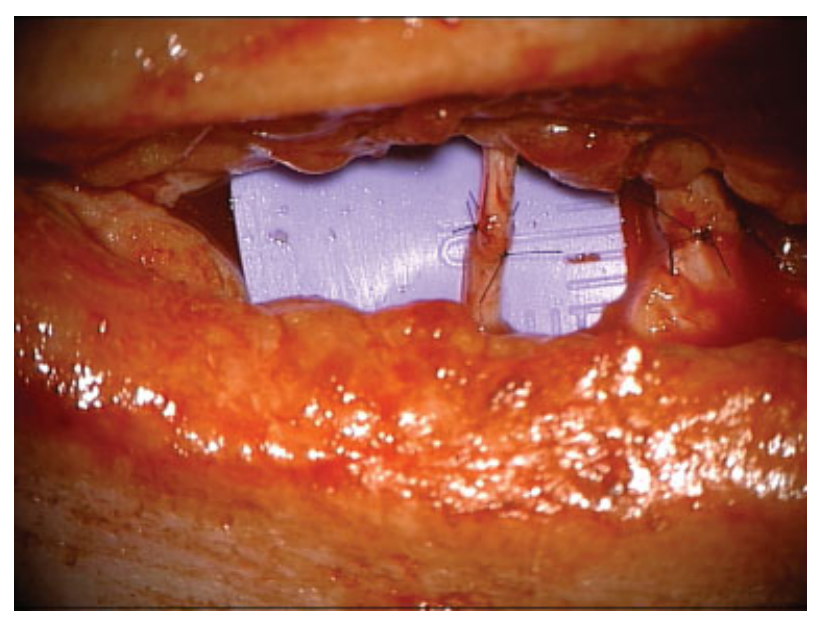

Fig. 13 Intraoperative view following primary neurorrhaphy of transected facial nerve (left) and re-anastomosis of transected parotid duct (right) over a stent due to a penetrating cheek injury.

risk of sialocele formation. ${ }^{26}$ Injection of botulinum toxin A into the gland (50-100 units) has proven to be more efficient and less uncomfortable in minimizing saliva secretion compared with restriction of oral intake or anti-sialogogue use. ${ }^{27}$ In cases of extensive glandular damage, subsequent surrounding edema may result in obstruction of the PD and possible sialadenitis. Therefore, the PD should be cannulated to maintain the patency of the lumen in these situations. ${ }^{26}$ It is advised that the canula be left in for 2 weeks in this scenario. ${ }^{28}$

\section{Parotid Duct Injury}

An initial attempt at diagnosing injury to the PD may be performed by massaging the parotid gland. Subsequent pooling of saliva in the surgical field is indicative of damage to the ductal structure. Alternatively, cannulating the distal opening of the duct with a pediatric catheter or lacrimal

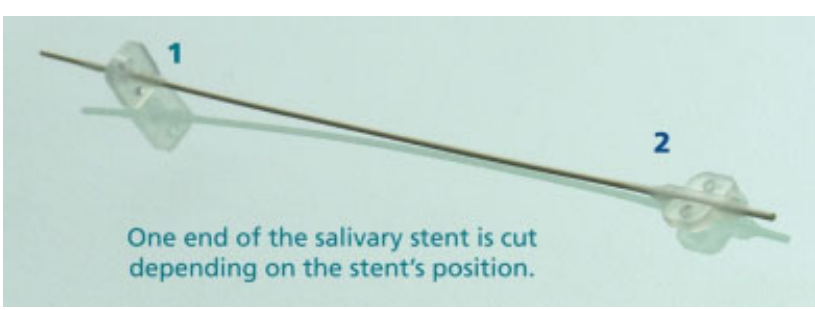

Fig. 14 Walvekar salivary duct stents have dual flanges that facilitate fixation to the buccal mucosa when used for the parotid duct (1) or to the floor of the mouth when used for the submandibular duct (2). probe followed by injection of saline may indicate ductal injury by the presence of saline from the distal severed duct within the surgical field. ${ }^{23}$ If there is no evidence of ductal compromise, it is recommended that the distal catheter be left in place for 1 week to prevent obstruction. ${ }^{26,28} \mathrm{~A}$ flexible sialendoscope may also be employed to locate the proximal and distal portions of the transected duct and allow for direct visualization of the ductal system. In cases of repair via reanastomosis, sialendoscopy is useful for assessment of the anastomosis both peri and postoperatively. ${ }^{29}$

When both stumps of the injured PD can be identified and the defect is less than $10 \mathrm{~mm}$, primary re-anastomosis is the preferred method of repair. ${ }^{26,27}$ Suturing over a cannula, introduced through the intraoral orifice, is thought to reduce the incidence of ductal stenosis. Venous, urethral, and epidural catheters have been used as stents. ${ }^{30-32}$ Öztürk et al described using an intubation tube cuff as a cannula. ${ }^{33}$ Silk or nylon splints have also been used to maintain duct integrity during suturing. 27,34

In our practice, we prefer the Walvekar Salivary stent (Hood Laboratories, Pembroke, MA), which is specifically designed and anatomically suited for use within the PD. It comes in sizes of 1.0,1.2, and 1.4-mm diameter, with the largest having the ability to be used as an irrigating catheter due to its luer-lock mechanism. It's defining feature is its dual flanges that are oriented for fixation to the floor of mouth (for submandibular duct cannulation) on one end and the buccal mucosa (for PD cannulation) on the other, preventing migration (-Fig. 14). Each stent comes with a calibrated guide wire that allows sizing of the length of the stent ( - Fig. 15). After insertion of the stent to span the transection, anastomosis over the stent may be performed with $7-0$ or $8-0$ nylon (-Fig. 16). It is secured to the mucosa using a loop stitch with a 4-0 nylon suture on a small cutting needle ( - Fig. 17). If using a stent for repair, it should be left in place for a minimum of 2 weeks to allow for healing, though we recommend leaving it in place for 4 to 6 weeks when placed for traumatic etiologies. If the ductal defect is greater than $1 \mathrm{~cm}$, reconstruction with an autologous facial vein graft has been performed successfully and may be considered. ${ }^{35,36}$

Damage to the distal portion of the duct (region C) may make identification of the severed ends of the duct more difficult and primary re-anastomosis may not be feasible. In this case, parotid diversion can be performed by transporting the proximal ductal stump through the buccinator muscle. The distal portion of the duct is then marsupialized to the buccal mucosa using 8-0 nylon suture, creating an artificial opening in the oral mucosa anterior to the natural papilla of the PD. ${ }^{26,37}$ It is advisable to leave a stent within the new orifice for 2 weeks to prevent stomal stenosis or obliteration. ${ }^{38}$

If there is extensive ductal trauma, making re-anastomosis or oral re-implantation impossible, the proximal end of the duct should be ligated or clamped. The resultant obstruction causes increased pressure within the parotid parenchyma, culminating in glandular atrophy. This process reduces the risk of sialocele and fistula formation..$^{20,23}$ Initially, swelling of the gland can be painful. Application of a pressure dressing can help promote atrophy. ${ }^{20}$ 


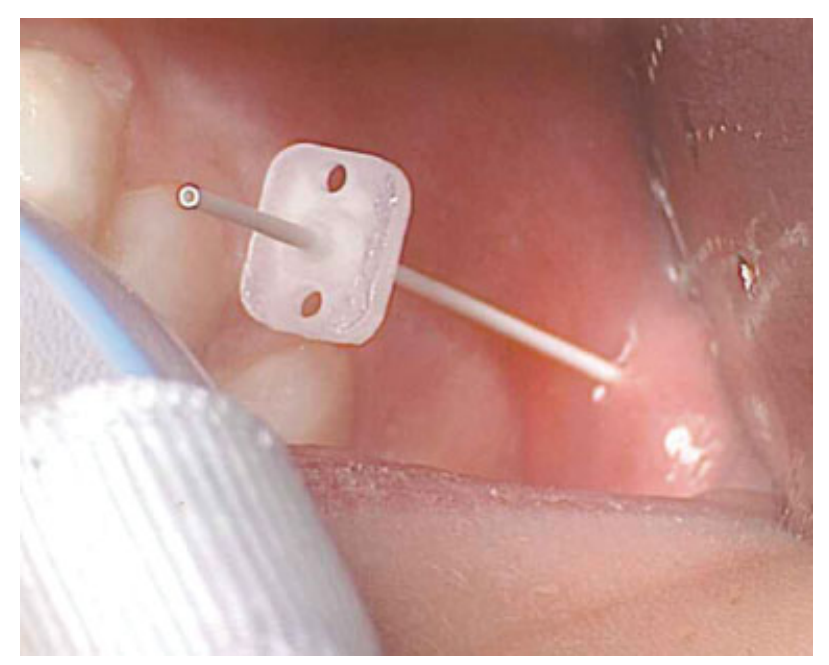

Fig. 15 Intraoperative view of Walvekar salivary duct stent after placement in the parotid duct and guidewire removed.

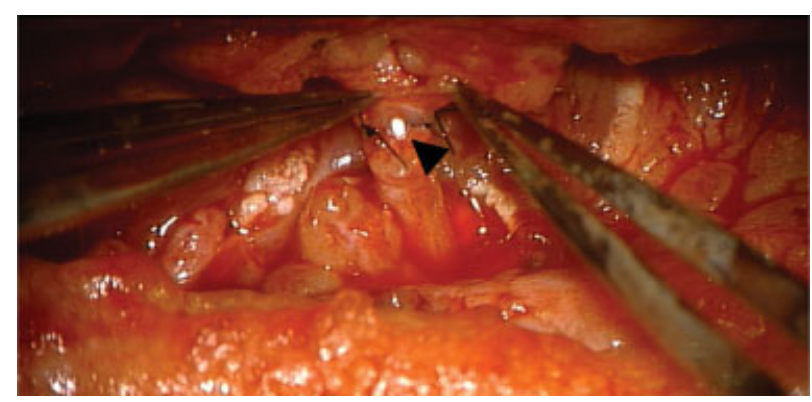

Fig. 16 Intraoperative view of Walvekar salivary duct stent (arrowhead) spanning re-anastomosed parotid duct.

\section{Complications}

\section{Stenosis}

Post-surgical stenosis of the PD, resulting in obstruction, often presents as recurrent painful swelling of the parotid gland following food intake. ${ }^{39}$ Most stenotic lesions occur in the proximal or middle third of the duct. In the past, conventional sialography has been used for diagnosis, but has mostly been abandoned due its need to be used with radiographic contrast. Ultrasound is a first-line diagnostic tool if stenosis is suspected. Alternatively, magnetic resonance sialography may be used. ${ }^{40}$ Sialendoscopy is the gold standard to establish final diagnosis and characterize the stenosis to plan accurate treatment. ${ }^{39}$ Depending on the severity of the stenosis, the current prevailing method of treatment involves a combination of ductal irrigation with a steroid solution (e.g., $16 \mathrm{mg}$ dexacort in isotonic saline) in conjunction with sialendoscopic-controlled dilation. For persistent stenosis, balloon dilation should be attempted. Ultimately, for high-grade stenosis, a stent may be kept in place for 4 to 6 weeks. $^{39,41}$

\section{Sialoceles and Fistulas}

A sialocele results from the accumulation of saliva in the tissues surrounding the parotid gland. It typically presents as a soft, tender, mobile mass in the region of the mandibular ramus approximately 8 to 14 days after initial injury. While sialoceles are often mistaken for hematomas, an aspirate with an amylase concentration above 10,000 U/L can confirm the diagnosis. ${ }^{26,42}$ Treatment depends on the time of sialocele presentation. In the case of an acute process, the wound should be surgically explored. Any damage to the parotid gland that is encountered should be cleaned and the parotid fascia should be sutured. ${ }^{28}$ If a lacerated duct is found, reapproximation is indicated. If unsuccessful, the proximal portion of the duct should be ligated and the sialocele should be drained intraorally via catheter. 28,37

Treatment of a late-forming sialocele consists of repeated percutaneous aspirations and pressure dressing application to induce glandular atrophy. ${ }^{26}$ Anti-sialagogues, such as glycopyrrolate ( $2 \mathrm{mg}$ twice daily) or scopolamine $(1.5 \mathrm{mg}$ transdermal patch every 48 hours), may be used as adjunctive therapy. ${ }^{43,44}$ In addition, antibiotics that cover Staph

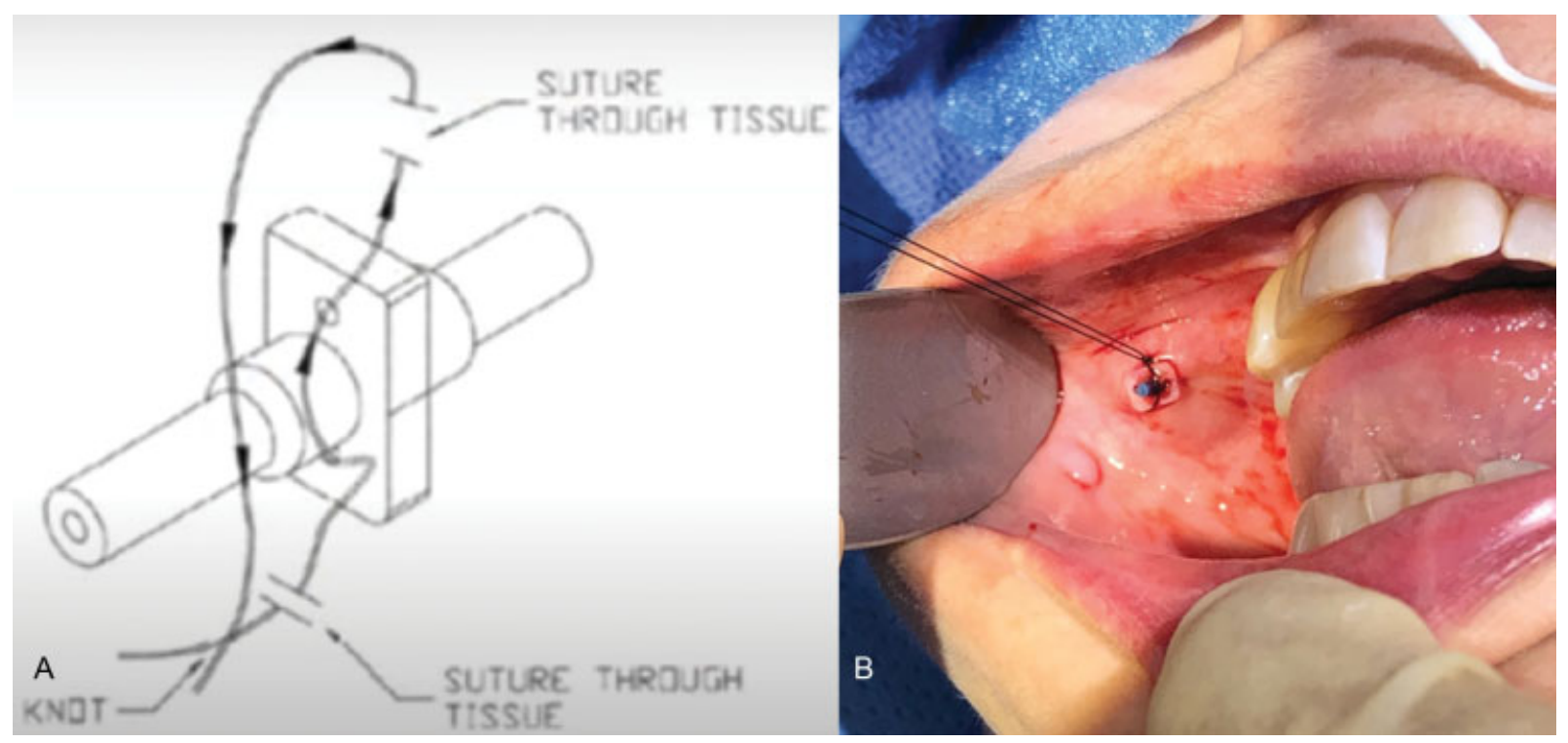

Fig. 17 Schematic (A) and in vivo photograph (B) of how to secure the Walvekar salivary duct stent flange to the mucosa using a loop stitch. 
Aureus, $H$. Influenzae, and gram-negative anaerobes may be considered (e.g., Augmentin $850 \mathrm{mg}$ BID). ${ }^{45}$ Some authors recommend parenteral nutrition to reduce salivation and allow for more rapid sialocele resolution. ${ }^{23,44}$

A fistula is a communication between the skin and the parotid gland that usually develops within the first week of injury. Sometimes a fistula may be due to a long-standing sialocele that ruptures the skin. Patients often notice clear secretions with milking of the gland and with mastication. If a fistula is an immediate complication, surgical re-exploration should be performed. ${ }^{26}$ Closure of the parotid capsule is indicated in the case of parotid gland laceration and primary re-anastomosis is preferred if ductal injury is encountered. The proximal portion of the duct should be ligated and secretions drained intraorally via catheter if re-anastomosis is not feasible. ${ }^{28}$ Treatment of a persistent fistula involves pressure dressings and anticholinergic medications. ${ }^{26,28}$

Several studies have shown intraglandular injections of botulinum toxin type $A$ to be effective in the treatment of both sialoceles and fistulas. ${ }^{46,47}$ Lovato et al described a technique used to achieve effective healing of post-parotidectomy fistula in which 10 to 20 units of botulinum toxin, fractionated into three doses, were injected transcutaneously into the parotid gland (two for the superior and one for the inferior lobe). Injections may be performed in conjunction with electromyographic monitoring to prevent undesirable weakening of the masseter and pterygoid muscles. Alternatively, ultrasound may be employed for guidance. Effects take place in 2 weeks and last between 2 to 3 months. ${ }^{46}$

If initial surgical and pharmacologic measures fail to resolve a sialocele or fistula, more aggressive interventions include radiation therapy or parotidectomy. Low-dose radiation acts to reduce glandular secretions by inducing fibrosis, though it has largely been abandoned due to the risk of malignancy. ${ }^{20,23}$ Tympanic neurectomy, which involves the transection of Jacobsen's nerve, a branch of the glossopharyngeal nerve, acts to reduce parasympathetic innervation to the parotid gland. ${ }^{47}$ Ultimately, superficial or total parotidectomy may be needed for refractory cases. ${ }^{34,37}$

\section{Conclusion}

Acute soft tissue trauma to the head and neck is among one of the most common reasons for emergency department presentation. Due to the risk of significant long-term functional and cosmetic compromise, evaluation of a patient who has suffered facial trauma should always include an FN exam and carry a low threshold of suspicion for PD injury. In FN repair, neurorrhaphy technique is primarily based on the ability to obtain tension-free anastomosis and outcomes are principally related to the timing of repair. PD injuries are generally repaired based on the site of ductal injury. Therefore, a fundamental knowledge of the anatomy of FN and PD and symptoms of their injuries is paramount in delivering optimal surgical results.

\section{Conflict of Interest}

None declared.

\section{References}

1 Birgfield C, Neligan P. Surgical approaches to facial nerve deficits. Skull Base 2011;21(03):177-184

2 Marks M, Polecritti D, Bergman R, Koch CA. Emergent soft tissue repair in facial trauma. Facial Plast Surg Clin North Am 2017;25 (04):593-604

3 Brown S, Isaacson B, Kutz W, Barnett S, Rozen S. Facial nerve trauma: clinical evaluation and management strategies. 2019; 143(05):1498-1512

4 Kannan RY, Hills A, Shelley MJ, et al. Immediate compared with late repair of extracranial branches of the facial nerve: a comparative study. Br J Oral Maxillofac Surg 2020;58(02): 163-169

5 Bascom D, Schaitkin B, May M, Klein S. Facial nerve repair: a retrospective review. Facial Plast Surg 2001;16(04):309-313

6 Ozmen OA, Falcioni M, Lauda L, Sanna M. Outcomes of facial nerve grafting in 155 cases: predictive value of history and preoperative function. Otol Neurotol 2011;32(08):1341-1346

7 Davies JC, Agur AM, Fattah A. Anatomic landmarks for localization of the branches of the facial nerve. OA Anat 2013;1(04):33

8 Altafulla J, Iwanaga J, Lachkar S, et al. The great auricular nerve: anatomical study with application to nerve grafting procedures. World Neurosurg 2019;125:e403-e407

9 Sural Nerve Graft Harvest. Iowa Head and Neck Protocols. June 2017. Accessed at https://medicine.uiowa.edu/iowaprotocols/ sural-nerve-graft-harvest. Feb 17, 2021

10 Wongkerdsook W, Agthong S, Amarase C, Yotnuengnit P, Huanmanop T, Chentanez V. Anatomy of the lateral antebrachial cutaneous nerve in relation to the lateral epicondyle and cephalic vein. Clin Anat 2011;24(01):56-61

11 Hoshal SG, Solis RN, Bewley AF. Nerve grafts in head and neck reconstruction. Curr Opin Otolaryngol 2020;28(05):346-351

12 Ray WZ, Mackinnon SE. Management of nerve gaps: autografts, allografts, nerve transfers, and end-to-side neurorrhaphy. Exp Neurol 2010;223(01):77-85

13 Lin MY, Manzano G, Gupta R. Nerve allografts and conduits in peripheral nerve repair. Hand Clin 2013;29(03):331-348

14 Brooks DN, Weber RV, Chao JD, et al. Processed nerve allografts for peripheral nerve reconstruction: a multicenter study of utilization and outcomes in sensory, mixed, and motor nerve reconstructions. Microsurgery 2012;32(01):1-14

15 Hu M, Xiao H, Niu Y, Liu H, Zhang L. Long-term follow-up of the repair of the multiple-branch facial nerve defect using acellular nerve allograft. J Oral Maxillofac Surg 2016;74(01):218. e1-218.e11

16 Azizzadeh B, Frisenda JL. Surgical management of postparalysis facial palsy and synkinesis. Otolaryngol Clin North Am 2018;51 (06):1169-1178

17 Markey JD, Loyo M. Latest advances in the management of facial synkinesis. Curr Opin Otolaryngol Head Neck Surg 2017;25(04): 265-272

18 Choi KH, Rho SH, Lee JM, Jeon JH, Park SY, Kim J. Botulinum toxin injection of both sides of the face to treat post-paralytic facial synkinesis. J Plast Reconstr Aesthet Surg 2013;66(08):1058-1063

19 Van Sickels JE. Parotid duct injuries. Oral Surg Oral Med Oral Pathol 1981;52(04):364-367

20 Lewis G, Knottenbelt JD. Parotid duct injury: is immediate surgical repair necessary? Injury 1991;22(05):407-409

21 Tachmes L, Woloszyn T, Marini C, et al. Parotid gland and facial nerve trauma: a retrospective review. J Trauma 1990;30(11): 1395-1398

22 Haller JR. Trauma to the salivary glands. Otolaryngol Clin North Am 1999;32(05):907-918

23 Pogrel MA, Schmidt B, Ammar A. The relationship of the buccal branch of the facial nerve to the parotid duct. J Oral Maxillofac Surg 1996;54(01):71-73

24 Robardey G, Le Roux MK, Foletti JM, et al. The Stensen's duct line: A landmark in parotid duct and gland injury and surgery. A 
prospective anatomical, clinical and radiological study. J Stomatol Oral Maxillofac Surg 2019;120(04):337-340

25 Lewkowicz AA, Hasson O, Nahlieli O. Traumatic injuries to the parotid gland and duct. J Oral Maxillofac Surg 2002;60(06): 676-680

26 Arnaud S, Batifol D, Goudot P, Yachouh J. Nonsurgical management of traumatic injuries of the parotid gland and duct using type a botulinum toxin. Plast Reconstr Surg 2006;117(07):2426-2430

27 Lazaridou M, Iliopoulos C, Antoniades K, Tilaveridis I, Dimitrakopoulos I, Lazaridis N. Salivary gland trauma: a review of diagnosis and treatment. Craniomaxillofac Trauma Reconstr 2012;5(04): 189-196

28 Koch M, Iro H, Bozzato A, Zenk J. Sialendoscopy-assisted microsurgical repair of traumatic transection of Stensen's duct. Laryngoscope 2013;123(12):3074-3077

29 Medeiros Paz ALL, Caetano RDS, Borges AH, Ricci Volpato LE. Reconstruction of the parotid duct. Ann Maxillofac Surg 2018;8 (01):140-142

30 Aloosi SN, Khoshnaw N, Ali SM, Muhammad BA. Surgical management of Stenson's duct injury by using double J stent urethral catheter. Int J Surg Case Rep 2015;17(01):75-78

31 Kumar SR, Hiremath V, Patil AG, Aparna S. Surgical management of Stenson's duct injury using epidural catheter: a novel technique. Niger J Clin Pract 2013;16(02):266-268

32 Öztürk MB, Barutca SA, Keskin ES, Atik B. Parotid duct repair with intubation tube: technical note. Ann Maxillofac Surg 2017;7(01): 129-131

33 Stevenson JH. Parotid duct transection associated with facial trauma: experience with 10 cases. Br J Plast Surg 1983;36(01):81-82

34 Colbert S, Haider F, Tan E, Brennan PA. Reconstruction of parotid duct using autologous vein graft and venous coupler. Br J Oral Maxillofac Surg 2013;51(03):273-274

35 Steinberg MJ, Herréra AF. Management of parotid duct injuries. Oral Surg Oral Med Oral Pathol Oral Radiol Endod 2005;99(02): 136-141
36 Gordin EA, Daniero JJ, Krein H, Boon MS. Parotid gland trauma. Facial Plast Surg 2010;26(06):504-510

37 Koch M, Iro H. Salivary duct stenosis: diagnosis and treatment. Acta Otorhinolaryngol Ital 2017;37(02):132-141

38 Ngu RK, Brown JE, Whaites EJ, Drage NA, Ng SY, Makdissi J. Salivary duct strictures: nature and incidence in benign salivary obstruction. Dentomaxillofac Radiol 2007;36(02):63-67

39 Ardekian L, Shamir D, Trabelsi M, Peled M. Chronic obstructive parotitis due to strictures of Stenson's duct-our treatment experience with sialoendoscopy. J Oral Maxillofac Surg 2010;68(01):83-87

40 Parekh D, Glezerson G, Stewart M, Esser J, Lawson HH. Posttraumatic parotid fistulae and sialoceles. A prospective study of conservative management in 51 cases. Ann Surg 1989;209(01): 105-111

41 Abramova L, Mann M, Hessler J, Sengelmann RD. Iatrogenic parotid sialocele after excision of malignant melanoma of the cheek. Dermatol Surg 2008;34(11):1584-1588

42 Lapid O, Kreiger Y, Sagi A. Transdermal scopolamine use for post-rhytidectomy sialocele. Aesthetic Plast Surg 2004;28(01): 24-28

43 Tandon P, Saluja H, Shah S, Dadhich A, Sachdeva S. Catheterization of post infection parotid duct sialocele with paediatric Ryles tube: A case report. J Oral Biol Craniofac Res 2018;8(03):217-220

44 Landau R, Stewart M. Conservative management of post-traumatic parotid fistulae and sialoceles: a prospective study. Br J Surg 1985;72(01):42-44

45 Lovato A, Restivo DA, Ottaviano G, Marioni G, Marchese-Ragona R. Botulinum toxin therapy: functional silencing of salivary disorders. Acta Otorhinolaryngol Ital 2017;37(02):168-171

46 Maharaj S, Mungul S, Laher A. Botulinum toxin A is an effective therapeutic tool for the management of parotid sialocele and fistula: A systematic review. Laryngoscope Investig Otolaryngol 2020;5(01):37-45

47 Vasama JP. Tympanic neurectomy and chronic parotitis. Acta Otolaryngol 2000;120(08):995-998 